\title{
Nagoya Termination Tool ${ }^{\star}$
}

\author{
Akihisa Yamada $^{1}$, Keiichirou Kusakari ${ }^{2}$, and Toshiki Sakabe ${ }^{1}$ \\ 1 Graduate School of Information Science, Nagoya University, Japan \\ 2 Faculty of Engineering, Gifu University, Japan
}

\begin{abstract}
This paper describes the implementation and techniques of the Nagoya Termination Tool, a termination prover for term rewrite systems. The main features of the tool are: the first implementation of the weighted path order which subsumes most of the existing reduction pairs, and the efficiency due to the strong cooperation with external SMT solvers. We present some new ideas that contribute to the efficiency and power of the tool.
\end{abstract}

\section{Introduction}

Proving termination of term rewrite systems (TRSs) has been an active field of research. In this paper, we describe the Nagoya Termination Tool (NaTT), a termination prover for TRS, which is available at

$$
\text { http://www.trs.cm.is.nagoya-u.ac.jp/NaTT/ }
$$

$\mathrm{NaTT}$ is powerful and fast; its power comes from the novel implementation of the weighted path order (WPO) [25, 26] that subsumes most of the existing reduction pairs, and its efficiency comes from the strong cooperation with stateof-the-art satisfiability modulo theory (SMT) solvers. In principle, any solver that complies with the SMT-LIB Standard $\sqrt{3}$ version 2.0 can be incorporated as a back-end into NaTT.

In the next section, we recall the dependency pair framework that NaTT is based on, and present existing techniques that are implemented in NaTT. Section 3 describes the implementation of WPO and demonstrates how to obtain other existing techniques as instances of WPO. Some techniques on cooperating with SMT solvers are presented in Section 4. After giving some design details in Section 5, we assess the tool by its results in the termination competition 4 in Section 6. Then we conclude in Section 7.

\section{The Dependency Pair Framework}

The overall procedure of NaTT is illustrated in Figure 1. NaTT is based on the

\footnotetext{
* Full version of the paper which is to appear in the Proceedings of the Joint 25th International Conference on Rewriting Techniques and Applications and 12th International Conference on Typed Lambda Calculi and Applications (RTA-TLCA '14), LNCS Advanced Research in Computing and Software Science, Springer, 2014.

3 http://www.smtlib.org/

4 http://termination-portal.org/wiki/Termination_Competition
} 


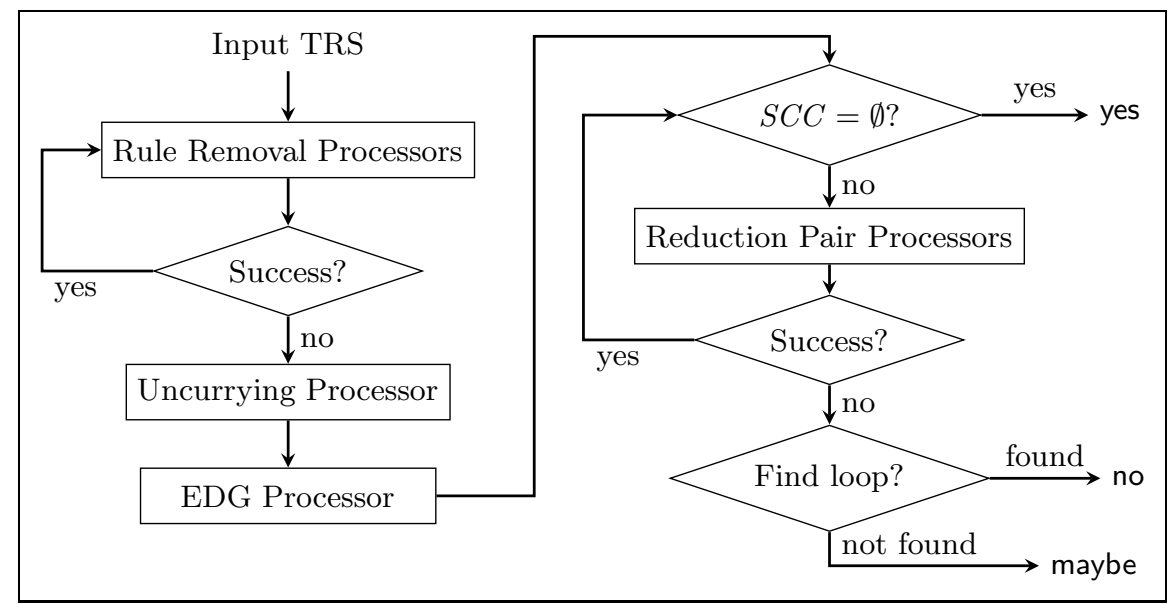

Fig. 1. Flowchart of NaTT

dependency pair framework (DP framework) [1,9, 10, a very successful technique for proving termination of TRSs which is implemented in almost all the modern termination provers for TRSs. In the DP framework, dependencies between function calls defined in a TRS $\mathcal{R}$ is expressed by the set $\operatorname{DP}(\mathcal{R})$ of dependency pairs. If a function $f$ is defined by a rule

$$
f\left(s_{1}, \ldots, s_{n}\right) \rightarrow C\left[g\left(t_{1}, \ldots, t_{m}\right)\right] \in \mathcal{R}
$$

where $g$ is also defined in $\mathcal{R}$, then this dependency is described by the following dependency pair:

$$
f^{\sharp}\left(s_{1}, \ldots, s_{n}\right) \rightarrow g^{\sharp}\left(t_{1}, \ldots, t_{m}\right) \in \operatorname{DP}(\mathcal{R})
$$

The DP framework (dis)proves termination of $\mathcal{R}$ by simplifying and decomposing $D P$ problems $\langle\mathcal{P}, \mathcal{R}\rangle$, where initially $\mathcal{P}=\mathrm{DP}(\mathcal{R})$. To this end, many $D P$ processors have been proposed. NaTT implements the following DP processors:

\subsection{Dependency Graph Processor}

This processor decomposes a DP problem $\langle\mathcal{P}, \mathcal{R}\rangle$ into $\left\langle\mathcal{P}_{1}, \mathcal{R}\right\rangle \ldots\left\langle\mathcal{P}_{n}, \mathcal{R}\right\rangle$ where $\mathcal{P}_{1}, \ldots, \mathcal{P}_{n}$ are the strongly connected components (SCCs) of the dependency graph 7, 10. Since the dependency graph is not computable in general, several approximations called estimated dependency graphs (EDGs) have been proposed. $\mathrm{NaTT}$ implements the EDG proposed in 8 .

\subsection{Reduction Pair Processor}

This processor forms the core of NaTT. A reduction pair is a pair $\langle\succsim, \succ\rangle$ of orders s.t. $\succ$ is compatible with $\succsim$ (i.e., $\succsim \cdot \succ \cdot \succsim \subseteq \succ$ ), both of $\succsim$ and $\succ$ are stable under 
substitution, $\succsim$ is monotone and $\succ$ is well-founded. From a DP problem $\langle\mathcal{P}, \mathcal{R}\rangle$, if all the involved rules are weakly decreasing (i.e., $\mathcal{P} \cup \mathcal{R} \subseteq \succsim$ ), strictly decreasing rules in $\mathcal{P}$ (w.r.t. $\succ$ ) can be removed. A great number of techniques for obtaining reduction pairs have been proposed so far. NaTT supports the following ones:

- Some simplification orders combined with argument filters [1]:

- the Knuth-Bendix order (KBO) [15] and its variants including KBO with status [20], the generalized $K B O$ [19] and the transfinite $K B O$ [18,22],

- the recursive path order [3] and the lexicographic path order (LPO) [14],

- polynomial interpretations (POLO) [1,17] and its variants, including certain forms 5 of POLO with negative constants [11] and max-POLO [6],

- the matrix interpretation method [4,13, and

- the weighted path order (WPO) [25,26].

Note that all of the above mentioned reduction pairs are subsumed by WPO. That is, by implementing WPO we obtain the other reduction pairs for free. We discuss the implementation details in Section 3 .

\subsection{Rule Removal Processor}

In the worst case, the size of dependency pairs is quadratic in the size of the input TRS $\mathcal{R}$. Hence it is preferable to reduce the size of $\mathcal{R}$ before computing dependency pairs. To this end NaTT applies the rule removal processor [7. If all rules in $\mathcal{R}$ are weakly decreasing w.r.t. a monotone reduction pair, then the processor removes strictly decreasing rules from $\mathcal{R}$. The required monotonicity of a reduction pair is obtained by choosing appropriate parameters for the implementation of WPO described above.

\subsection{Uncurrying Processor}

Use of uncurrying for proving termination is proposed for applicative rewrite systems in [12. The uncurrying implemented in NaTT is similar to the generalized version proposed in 21, in the sense that it does not assume application symbols to be binary. A symbol $f$ is considered as an application symbol if all the following conditions hold:

- $f$ is defined and has positive arity,

- a subterm of the form $f(x, \ldots)$ does not occur in any left-hand-sides of $\mathcal{R}$,

- a subterm of the form $f(g(\ldots), \ldots)$ occurs in some right-hand-side of $\mathcal{R}$.

If such application symbols are found, then $\mathcal{R}$ is uncurried w.r.t. the uncurrying TRS $\mathcal{U}$ that consists of the following rules 6

$$
f\left(f^{l} g\left(x_{1}, \ldots, x_{m}\right), y_{1}, \ldots, y_{n}\right) \rightarrow f^{l+1} g\left(x_{1}, \ldots, x_{m}, y_{1}, \ldots, y_{n}\right)
$$

for every $g \neq f$ and $l$ less than the applicative arity 7 of $g$, where $f^{0} g$ denotes $g$ and $f^{l+1} g$ is a new function symbol of arity $m+n$.

\footnotetext{
${ }^{5}$ Here, negative values are allowed only for the constant part.

6 The notation is derived from the freezing technique [23].

${ }^{7}$ Applicative arities are taken so that $\eta$-saturation is not needed.
} 


\section{The Weighted Path Order}

As we mentioned in the introduction, NaTT implements only WPO for obtaining reduction pairs. WPO is parameterized by (1) a weight algebra which specifies how weights are computed, (2) a precedence on function symbols, and (3) a status function which specifies how arguments are compared. In the following sections, we present some options which NaTT provides for specifying search spaces for these parameters.

\subsection{Templates for Weight Algebras}

One of the most important tasks in proving termination by WPO is finding an appropriate weight algebra. In order to reduce the task to an SMT problem, NaTT considers template algebras over integers. Currently the following template algebras are implemented:

- The algebra $\mathcal{P}$ ol indicates that weights of terms are computed by a linear polynomial. Interpretations are in the following shape:

$$
f_{\mathcal{P o l}}\left(x_{1}, \ldots, x_{n}\right)=w_{f}+\sum_{i=1}^{n} c_{f, i} \cdot x_{i}
$$

where the template variables $w_{f}$ and $c_{f, 1}, \ldots, c_{f, n}$ should be decided by an external SMT solver.

- The algebra Max indicates that weights are computed using the max operator. A symbol $f$ with arity $\geq 1$ is interpreted in the following shape:

$$
f_{\mathcal{M a x}}\left(x_{1}, \ldots, x_{n}\right)=\max _{i=1}^{n}\left(p_{f, i}+c_{f, i} \cdot x_{i}\right)
$$

where $p_{f, 1}, \ldots, p_{f, n}$ are template variables. For constant symbols, interpretations of the shape (10) are used. Since the operator max is not usually supported by SMT solvers, these interpretations are encoded as quantifierfree formulas using the technique presented in [25].

- The algebra $\mathcal{M P o l}$ combines both forms of interpretations described above. Since it is inefficient to consider all combinations of these interpretations, $\mathcal{M P o l}$ decides the shape of interpretations according to the following intuition: If a constraint such as $f(x)>g(x, x)$ appears, then $g$ is interpreted as $g_{\mathcal{M a x}}$, because the imposed constraint $c_{f, 1} \geq c_{g, 1} \wedge c_{f, 1} \geq c_{g, 2}$ is easier than $c_{f, 1} \geq c_{g, 1}+c_{g, 2}$, which would be imposed by the interpretation $g_{\mathcal{P o l}}$.

The template variables introduced above are partitioned into two groups: template variables $w_{f}, p_{f, 1}, \ldots, p_{f, n}$ are grouped in the constant part, and tem-

plate variables $c_{f, 1}, \ldots, c_{f, n}$ are in the coefficient part. For efficiency, it is important to properly restrict the range of these variables. 
Table 1. Parameters for some monotone reduction pairs.

\begin{tabular}{|c|c|c|c|c|c|}
\hline Technique & \multicolumn{5}{|c|}{ template coefficient constant precedence status } \\
\hline Linear POLO & Pol & $\mathbb{Z}_{+}$ & $\mathbb{N}$ & no & empty \\
\hline LPO & $\operatorname{Max}$ & $\{1\}$ & $\{0\}$ & yes & total \\
\hline $\mathrm{KBO} 8$ & $\mathcal{P} o l$ & $\{1\}$ & $\mathbb{N}$ & yes & total \\
\hline Transfinite $\mathrm{KBO}^{8}$ & Pol & $\mathbb{Z}_{+}$ & $\mathbb{N}$ & yes & total \\
\hline
\end{tabular}

\subsection{Classes of Precedences}

NaTT offers "quasi" and "strict" precedences, as well as an option to disable them (i.e., all symbols are considered to have the same precedence). For reduction pairs using precedences, we recommend quasi-precedences which are chosen by default, as the encoding follows the technique of [27] that naturally encodes quasi-precedences.

\subsection{Classes of Status Functions}

NaTT offers three classes of status functions: "total", "partial" and "empty" ones. The standard notions of status functions are total ones that were introduced to admit permutation of arguments when comparing them lexicographically from left to right (cf. 20]). Such a comparison appears in many well-known reduction pairs; famous examples are LPO and KBO. By combining the idea of argument filters, status functions have recently been generalized to partial ones, that do not only permute but may also drop some arguments 24]. A partial status is beneficial for KBO, and even more significant when combined with WPO [26]. The extreme case of a partial status is the "empty" status, that drops all arguments and so no comparison of arguments will be performed. This option corresponds to the nature of interpretation methods, e.g. POLO, if precedences are also disabled.

\subsection{Obtaining Well-known Reduction Pairs}

Although most of the existing reduction pairs are subsumed by WPO, some of them are still useful for improving efficiency, due to the restricted search space and simplified SMT encoding. We list parameters that correspond to some known reduction pairs in Tables 1 and 2. Note here that the effects of non-collapsing argument filters are simulated by allowing 0-coefficients in the weight algebra. Thus NaTT has a dedicated implementation only for collapsing argument filters, and implementations of usable rules for interpretation methods and path orders are smoothly unified.

\footnotetext{
${ }^{8}$ Further constraints for admissibility are imposed.
} 
Table 2. Parameters for some (non-monotone) reduction pairs.

\begin{tabular}{l|ccccc} 
Technique & \multicolumn{4}{|c}{ template coefficient } & constant precedence status \\
\hline Linear POLO & $\mathcal{P}$ ol & $\mathbb{N}$ & $\mathbb{N}$ & no & empty \\
Max-POLO & $\mathcal{M}$ Pol & $\mathbb{N}$ & $\mathbb{Z}$ & no & empty \\
LPO + argument filter & $\mathcal{M} a x$ & $\{0,1\}$ & $\{0\}$ & yes & total \\
KBO + argument filter & $\mathcal{P} o l$ & $\{0,1\}$ & $\mathbb{N}$ & yes & total \\
Matrix interpretations & $\mathcal{P} o l$ & $\mathbb{N}^{d \times d}$ & $\mathbb{N}^{d}$ & no & empty \\
WPO $(\mathcal{M S}$ sum $)$ & $\mathcal{M}$ Pol & $\{0,1\}$ & $\mathbb{N}$ & yes & partial
\end{tabular}

\section{Cooperation with SMT Solvers}

$\mathrm{NaTT}$ is designed to work with any SMT-LIB 2.0 compliant solvers that support at least QF_LIA logic, for which various efficient solvers exist.9 NaTT extensively uses SMT encoding techniques for finding appropriate reduction pairs; the conditions of reduction pair processors are encoded into the following SMT constraint:

$$
\bigwedge_{l \rightarrow r \in \mathcal{R}} \llbracket l \succsim r \rrbracket \wedge \bigwedge_{s \rightarrow t \in \mathcal{P}} \llbracket s \succsim t \rrbracket \wedge \bigvee_{s \rightarrow t \in \mathcal{P}} \llbracket s \succ t \rrbracket
$$

where each $\llbracket l(r \rrbracket$ is an SMT formula that represents the condition $l(r$. In the remainder of this section, we present two techniques for handling such constraints that contribute to the efficiency of $\mathrm{NaTT}$.

\subsection{Use of Interactive Features of SMT Solvers}

In a typical run of termination verification, constraints of the form (3) are generated and solved many times, and each encoding sometimes involves thousands of lines of SMT queries with a number of template and auxiliary variables. Hence runtime spent for the SMT solver forms a large part of the overall runtime of the tool execution. NaTT tries to reduce the runtime by using interactive features of SMT solvers 10 which are specified in SMT-LIB 2.0.

For each technique of reduction pairs, the encoded formula of the constraint $\bigwedge_{l \rightarrow r \in \mathcal{R}} \llbracket l \succsim r \rrbracket$ need not be changed during a run, as far as $\mathcal{R}$ is not modified 11 Hence, when a reduction pair processor is applied for the first time, the back-end SMT solver is initialized according to the following pseudo-script:

$$
\begin{aligned}
& \text { (assert } \left.\left(\bigwedge_{l \rightarrow r \in \mathcal{R}}\left(u_{l \rightarrow r} \Rightarrow \llbracket l \succsim r \rrbracket\right)\right)\right) \\
& \text { (push) }
\end{aligned}
$$

${ }^{9}$ Cf. the Satisfiability Modulo Theories Competition, http://smtcomp.org/.

10 NaTT is not the first tool to use the interactive features of SMT solvers. For example, the Houdini implementation in Boogie uses the features [16.

${ }^{11}$ Although rules in $\mathcal{R}$ may be removed by considering usable rules, the formula still need not be changed, since it can be simulated by negating a propositional variable that represents whether the rule is usable or not. 
where $u_{l \rightarrow r}$ is a boolean variable denoting whether the rule $l \rightarrow r$ is usable or not. When the processor is applied to an SCC $\mathcal{P}$, the following script is used:

$$
\begin{aligned}
& \left(\operatorname{assert}\left(\bigwedge_{s \rightarrow t \in \mathcal{P}} \llbracket s \succsim t \rrbracket \wedge \bigvee_{s \rightarrow t \in \mathcal{P}} \llbracket s \succ t \rrbracket\right)\right) \\
& (\text { check-sat) }
\end{aligned}
$$

Then, if a solution is found by the SMT solver, NaTT analyzes the solution using the get-value command. After this analysis, the command

$$
\text { (pop) }
$$

is issued to clear the constraints due to $\mathcal{P}$ and go back to the context saved by the (push) command. In order to derive the best performance of the solver,

\section{(reset)}

is also issued in case sufficiently many rules become unusable (e.g., 1/3 of the rules in $\mathcal{R}$ ) from $\mathcal{P}$. All these commands, push, pop and reset are expected to be available in SMT-LIB 2.0 compliant solvers.

\subsection{Use of Linear Arithmetic}

Note that expressions of the form (11) or (2) are nonlinear, due to the coefficients $c_{f, 1}, \ldots, c_{f, n}$. However, not many SMT solvers support nonlinear arithmetic, and even if they do, they are much less scalable than they are for linear arithmetic. Hence, we consider reducing the formulas to linear ones by restricting the range of $c_{f, 1}, \ldots, c_{f, n}$ e.g. to $\{0,1\}$. Although the idea is inspired by [2], NaTT uses a more straightforward reduction using ite (if-then-else) expressions. Each coefficient $c_{f, i}$ is replaced by the expression (ite $b_{f, i} 10$ ) where $b_{f, i}$ is a propositional variable, and then multiplications are reduced according to the rule:

$$
\left(*\left(\text { ite } e_{1} e_{2} e_{3}\right) e_{4}\right) \rightarrow\left(\text { ite } e_{1}\left(* e_{2} e_{4}\right)\left(* e_{3} e_{4}\right)\right)
$$

It is easy to see that this reduction terminates and linearizes expressions of the form (1) or (2). It is also possible to avoid an explosion of the size of formulas by introducing a auxiliary variable for the duplicated expression $e_{4}$.

Example 1. Consider the constraint $\mathrm{f}(\mathrm{f}(\mathrm{a}))>\mathrm{b}$ interpreted in the algebra Pol, and suppose that the range of $c_{\mathrm{f}, 1}$ is restricted to $\{1,2\}$. The interpretation of the term $f(f(a))$ is reduced as follows (written as S-expressions):

$$
\begin{aligned}
\llbracket \mathrm{f}(\mathrm{f}(\mathrm{a})) \rrbracket & \left.=\left(+w_{\mathrm{f}} \quad\left(* \quad \text { ite } b_{\mathrm{f}, 1} \quad 2 \quad 1\right) \llbracket \mathrm{f}(\mathrm{a}) \rrbracket\right)\right) \\
& \rightarrow\left(+w_{\mathrm{f}} \quad\left(\text { ite } b_{\mathrm{f}, 1} \quad(* 2 \quad \llbracket \mathrm{f}(\mathrm{a}) \rrbracket) \llbracket \mathrm{f}(\mathrm{a}) \rrbracket\right)\right)
\end{aligned}
$$

Similarly, for $f(a)$ we obtain

$$
\llbracket \mathrm{f}(\mathrm{a}) \rrbracket \rightarrow\left(+w_{\mathrm{f}} \quad\left(\text { ite } b_{\mathrm{f}, 1} \quad\left(* 2 w_{\mathrm{a}}\right) w_{\mathrm{a}}\right)\right)
$$

Now, the constraint $\llbracket f(f(a))>b \rrbracket$ is expressed by the following script: 


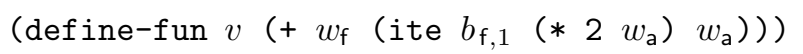

In contrast to SAT encoding techniques [4] 6 , we do not have to care about the bit-width for the constant part and intermediate results. It is also possible to indicate that NaTT should keep formulas nonlinear, and solve them using SMT solvers that support QF_NIA logic. Our experiments on TPDB 12 problems, however, suggests that use of nonlinear SMT solving is impractical for our purpose.

\section{Design}

The source code of NaTT consists of about 6000 lines of code written in OCaml 13 About $23 \%$ is consumed by interfacing SMT solvers, where some optimizations for encodings are also implemented. Another $17 \%$ is for parsing command-lines and TRS files. The most important part of the source code is the $40 \%$ devoted to the implementation of WPO, the unified reduction pair processor. Each of the other processors implemented consumes less than 3\%. For computing SCCs, the third-party library ocamlgraph 14 is used.

\subsection{Command Line Interface}

The command line of NaTT has the following syntax:

$$
. / \text { NaTt }[\text { FILE] [OPTION] ... [PROCESSOR] ... }
$$

To execute NaTT, an SMT-LIB 2.0 compliant solver must be installed. By default, z3 version 4.0 or later 15 is supposed to be installed in the path. Users can specify other solvers by the --smt "COMMAND" option, where the solver invoked by COMMAND should process SMT-LIB 2.0 scripts given on the standard input.

The TRS whose termination should be verified is read from either the specified FILE or the standard input 16 Each PROCESSOR is either an order (e.g. POLO, KBO, WPO, etc., possibly followed by options), or a name of other processors (UNCURRY, EDG, or LOOP). Orders preceding the EDG processor should be monotone reduction pairs and applied as rule removal processors before computing the dependency pairs. Orders following the EDG processor are applied as reduction pair processors to each SCC in the EDG. A list of available OPTIONs and PROCESSORs can be obtained via NaTT --help.

$\overline{12}$ The Termination Problem Data Base, http://termination-portal.org/wiki/TPDB.

13 http://caml.inria.fr/

14 http://ocamlgraph.lri.fr/

15 http://z3.codeplex.com/

16 The format is found at https://www.lri.fr/ marche/tpdb/format.html. 
Table 3. Effects of the optimizations.

\begin{tabular}{cccccc}
\hline option & yes & no & maybe & T.O. & time \\
\hline non-linear & 767 & 170 & 368 & 158 & 13138.11 \\
linearized & 848 & 173 & 429 & 13 & 2161.43 \\
interactive & 848 & 173 & 429 & 13 & 1865.50 \\
\hline
\end{tabular}

\subsection{The Default Strategy}

In case no PROCESSOR is specified, the following default strategy will be applied:

- As a rule removal processor, POLO with coefficients in $\{1,2\}$ and constants in $\mathbb{N}$ is applied.

- Then the uncurrying processor is applied.

- The following reduction pair processors are applied (in this order):

1. POLO with coefficients in $\{0,1\}$ and constants in $\mathbb{N}$,

2. algebra Max with coefficients in $\{0,1\}$ and constants in $\mathbb{N}$,

3. LPO with quasi-precedence, status and argument filter,

4. algebra $\mathcal{M P o l}$ with coefficients in $\{0,1\}$ and constants in $\mathbb{Z}$,

5. WPO with quasi-precedence, partial status, algebra $\mathcal{M P o l}$, coefficients in $\{0,1\}$ and constants in $\mathbb{N}$,

6. matrix interpretations with $\{0,1\}^{2 \times 2}$ matrices and $\mathbb{N}^{2}$ vectors.

- If all the above processors fail, then a (naive) loop detection is performed.

\section{Assessment}

In this section, we verify the significance of the contributions of NaTT by experiments and by its result in the termination competition.

\subsection{Effects of Optimizations}

First, we verify the effect of the optimizations proposed in Section 4 . The experiments are run on a server equipped with a quad-core Intel Xeon E5-3407v2 processor running at a clock rate of $2.40 \mathrm{GHz}$ and $32 \mathrm{~GB}$ of main memory. As the SMT solver, we choose z3 4.3.2.

In Table 3, we compare the following options of NaTT.

- The 'non-linear' row considers interpretations of the non-linear shape of (1) and (2), and directly solves the encoded problem via QF-NIA logic. To achieve a practical runtime, the constant part is bounded by upper bound 3 .

- The 'linearized' row applies the linearization proposed in Section 4.2

- The 'interactive' row further uses the interactive features as proposed in Section 4.1. This option is the default of NaTT.

In the table, we observe a dramatic improvement by the linearization of Section 4.2. The use of interactive features of SMT solvers may look less significant, but the runtime improves by almost $10 \%$. 


\subsection{Results in the Termination Competition}

Many tools have been developed for proving termination of TRSs, and the international termination competition has been held annually for a decade. NaTT participated in the TRS Standard category of the full-run 2013 17 where the other participants are versions of: AProVE $18 \mathrm{~T}_{\mathrm{T}} \mathrm{T}_{2}, 19 \mathrm{MU}$-TERM 20 and WANDA 21 Using the default strategy described in Section 5.2. NaTT (dis)proves termination of 982 TRSs (unfortunately, the competition version of NaTT failed to input 36 problems due to a bug in parser) and comes next to (the two versions of) AProVE, the constant champion of the category. It should be noticed that NaTT proved termination of 34 TRSs out of the 159 whose termination could not be proved by any other tool. NaTT is notably faster than the other competitors; it consumed only $21 \%$ of the time compared to AProVE, the second fastest. We expect that we can further improve efficiency by optimizing to multi-core architecture; currently, NaTT runs in almost single thread.

NaTT also participated in the SRS Standard category. However, the result is not as good as it is for TRSs. This is due to the fact that the default strategy of Section 5.2 is designed only for non-unary signatures. Indeed, when a unary symbol is considered, an interpretation of the form (2) is equivalent to one of the form (11), It should be improved by choosing a strategy depending on the shape of input TRSs.

\section{Conclusion}

We described the implementation and techniques of the termination tool NaTT. The novel implementation of the weighted path order is described in detail, and some techniques for cooperating SMT solvers are presented. Together with these efforts, NaTT is one of the most efficient and strongest tools for proving termination of TRSs.

Because of its efficiency, NaTT is especially strong on larger systems. In general, a larger input TRS requires a larger proof script to be produced, which is quite difficult to be checked by hand. Thus our future work is to produce proofs in the certifiable proof format 22

Acknowledgments We thank the anonymous reviewers of this paper for careful inspections and constructive comments that improved the quality of this paper. We also thank Shaz Qadeer for information about Boogie. This work was supported by JSPS KAKENHI \#24500012.

17 http://termcomp.uibk.ac.at/

18 http://aprove.informatik.rwth-aachen.de/

19 http://cl-informatik.uibk.ac .at/software/ttt2/

20 http://zenon.dsic.upv.es/muterm/

21 http://wandahot.sourceforge.net/

22 http://cl-informatik.uibk.ac.at/software/cpf/ 


\section{References}

1. T. Arts and J. Giesl. Termination of term rewriting using dependency pairs. Theor. Compt. Sci., 236(1-2):133-178, 2000.

2. C. Borralleras, S. Lucas, R. Navarro-Marset, E. Rodríguez-Carbonell, and A. Rubio. Solving non-linear polynomial arithmetic via SAT modulo linear arithmetic. In Proc. CADE' '09, LNCS 5663, pages 294-305, 2009.

3. N. Dershowitz. Orderings for term-rewriting systems. Theor. Compt. Sci., 17(3):279-301, 1982.

4. J. Endrullis, J. Waldmann, and H. Zantema. Matrix interpretations for proving termination of term rewriting. J. Autom. Reasoning, 40(2-3):195-220, 2008.

5. C. Fuhs, J. Giesl, A. Middeldorp, P. Schneider-Kamp, R. Thiemann, and H. Zankl. SAT solving for termination analysis with polynomial interpretations. In Proc. SAT '0\%, LNCS 4501, pages 340-354, 2007.

6. C. Fuhs, J. Giesl, A. Middeldorp, P. Schneider-Kamp, R. Thiemann, and H. Zankl. Maximal termination. In Proc. RTA '08, LNCS 5117, pages 110-125, 2008.

7. J. Giesl, R. Thiemann, and P. Schneider-Kamp. The dependency pair framework: Combining techniques for automated termination proofs. In Proc. LPAR '04, LNAI 3452, pages 75-90, 2004.

8. J. Giesl, R. Thiemann, and P. Schneider-Kamp. Proving and disproving termination of higher-order functions. In Proc. FroCoS '05, LNAI 3717, pages 216-231, 2005.

9. J. Giesl, R. Thiemann, P. Schneider-Kamp, and S. Falke. Mechanizing and improving dependency pairs. J. Autom. Reasoning, 37(3):155-203, 2006.

10. N. Hirokawa and A. Middeldorp. Dependency pairs revisited. In Proc. RTA '04, LNCS 3091, pages 249-268, 2004.

11. N. Hirokawa and A. Middeldorp. Polynomial interpretations with negative coefficients. In Proc. AISC'04, LNAI 3249, pages 185-198, 2004.

12. N. Hirokawa, A. Middeldorp, and H. Zankl. Uncurrying for termination and complexity. J. Autom. Reasoning, 50(3):279-315, 2013.

13. D. Hofbauer and J. Waldmann. Termination of string rewriting with matrix interpretations. In Proc. RTA '06, LNCS 4098, pages 328-342, 2006.

14. S. Kamin and J.-J. Lévy. Two generalizations of the recursive path ordering, 1980. Unpublished note.

15. D.E. Knuth and P. Bendix. Simple word problems in universal algebras. In Computational Problems in Abstract Algebra, pages 263-297. Pergamon Press, New York, 1970 .

16. A. Lal, S. Qadeer, and S. Lahiri. Corral: A solver for reachability modulo theories. In Proc. CAV'12, LNCS 7358, pages 427-443, 2012.

17. D. Lankford. On proving term rewrite systems are Noetherian. Technical Report MTP-3, Louisiana Technical University, 1979.

18. M. Ludwig and U. Waldmann. An extension of the Knuth-Bendix ordering with LPO-like properties. In Proc. LPAR '07, LNAI 4790, pages 348-362, 2007.

19. A. Middeldorp and H. Zantema. Simple termination of rewrite systems. Theor. Compt. Sci., 175(1):127-158, 1997.

20. J. Steinbach. Extensions and comparison of simplification orders. In Proc. RTA '89, LNCS 355, pages 434-448, 1989.

21. C. Sternagel and R. Thiemann. Generalized and formalized uncurrying. In Proc. FroCoS'11, LNAI 6989, pages 243-258, 2011. 
22. S. Winkler, H. Zankl, and A. Middeldorp. Ordinals and Knuth-Bendix orders. In Proc. LPAR'12, LNCS ARCoSS 7180, pages 420-434, 2012.

23. H. Xi. Towards automated termination proofs through "freezing". In Proc. RTA '98, LNCS 1379, pages 271-285, 1998.

24. A. Yamada, K. Kusakari, and T. Sakabe. Partial status for KBO. In Proc. WST '13, pages 74-78, 2013.

25. A. Yamada, K. Kusakari, and T. Sakabe. Unifying the Knuth-Bendix, recursive path and polynomial orders. In Proc. PPDP'13, pages 181-192, 2013.

26. A. Yamada, K. Kusakari, and T. Sakabe. A unified order for termination proving. CoRR, abs/1404.6245, 2014. Submitted to SCP.

27. H. Zankl, N. Hirokawa, and A. Middeldorp. KBO orientability. J. Autom. Reasoning, 43(2):173-201, 2009. 